\title{
Dexametasona Comparada à Metoclopramida na Profilaxia de Vômitos Pós-Operatórios em Crianças Submetidas a Procedimentos Cirúrgicos Ambulatoriais *
}

\section{Dexamethasone Compared to Metoclopramide in the Prophylaxis of Emesis in Children Undergoing Ambulatory Surgical Procedures}

Antonio Bedin, TSA $^{1}$; Mauro de Souza Leite Pinho ${ }^{2}$; Cladir Terezinha Zanotelli ${ }^{3}$; Ângela Silvia Caldart ${ }^{4}$; Jurandir Coan Turazzi, TSA ${ }^{5}$; Renato Almeida Couto de Castro, TSA ${ }^{6}$

\section{RESUMO}

Bedin A, Pinho MSL, Zanotelli CT, Caldart AS, Turazzi JC, Castro RAC - Dexametasona Comparada à Metoclopramida na Profilaxia de Vômitos Pós-Operatórios em Crianças Submetidas a Procedimentos Cirúrgicos Ambulatoriais

JUSTIFICATIVA E OBJETIVOS: O vômito pós-operatório é uma complicação comum e desagradável. Porém, atualmente modelos matemáticos tais como número necessário para tratar (NNT) e redução do risco relativo (RRR) têm sido úteis para a decisão de que medicação utilizar para a profilaxia. O objetivo deste estudo foi verificar se a dexametasona, comparada à metoclopramida, reduz a incidência de vômitos, quando administrada por via venosa em crianças anestesiadas com sevoflurano em cirurgias pediátricas ambulatoriais.

MÉTODO: Participaram do estudo 237 crianças, do sexo masculino, com idade entre 11 meses e 12 anos, estado físico ASA I e II, submetidas à herniorrafia inguinal. Como medicação pré-anestésica foi utilizado midazolam por via oral. Para a indução e manutenção da anestesia foram utilizados sevoflurano, óxido nitroso e fentanil $\left(1 \mu \mathrm{g} \cdot \mathrm{kg}^{-1}\right)$. Os pacientes foram divididos em grupo $D(n=118)$ dexametasona $(150$ $\left.\mu \mathrm{g} \cdot \mathrm{kg}^{-1}\right)$ por via venosa na indução e grupo $M(n=119)$ metoclopramida $\left(150 \mu \mathrm{g} \cdot \mathrm{kg}^{-1}\right)$ na indução. Foram analisadas as incidências de vômitos nas primeiras 4 horas de pós-operatório (PO), entre 4 horas e 24 horas de PO, o NNT de ambas as medicações utilizadas e a $R R R$ da dexametasona em relação à metoclopramida.

RESULTADOS: A incidência de vômitos foi $9,32 \%$ no grupo $D$ e $33,61 \%$ no grupo $M$ durante as primeiras 4 horas de $P O$ e

\footnotetext{
* Recebido do (Received from) Hospital Municipal São José, Joinville, $S C$

1. Anestesiologista. Co-Responsável pelo CET/SBA de Joinville

2. Coloproctologista. Doutorado em Medicina pela Universidade de Birmingham, Inglaterra. Docente do Programa de Mestrado em Saúde e Meio Ambiente da Univille

3. Graduada em Matemática. Doutorado em Engenharia de Produção, Universidade Federal de Santa Catarina. Docente do Programa de Mestrado em Saúde e Meio Ambiente da Univille

4. Graduada em Farmácia e Bioquímica. Farmacêutica da Secretaria Municipal de Saúde de Joinville

5. Anestesiologista. Co-responsável pelo CET/SBA de Joinville. Coordenador do Serviço de Anestesiologia de Joinville

6. Anestesiologista. Responsável pelo CET/SBA de Joinville

Apresentado (Submitted) em 13 de setembro de 2004

Aceito (Accepted) para publicação em 14 de março de 2005

Endereço para Correspondência (Correspondence to)

Dr. Antonio Bedin

R. Doutor Roberto Koch, 72 Bairro Atiradores

89203-088 Joinville. SC

E-mail: abjoi@uol.com.br
}

(c) Sociedade Brasileira de Anestesiologia, 2005

$1,69 \%$ com a dexametasona e de 3,36\% com a metoclopramida entre 4 e 24 horas de PO. O RRR foi $72 \%$ da dexametasona em relação à metoclopramida nas primeiras 4 horas. O NNT da dexametasona foi 3,25 e o da metoclopramida foi 15,66.

CONCLUSÕES: A dexametasona reduz, de forma mais eficiente que a metoclopramida, a incidência de vômitos quando utilizada durante a indução de anestesia com sevoflurano associado ao óxido nitroso e fentanil.

Unitermos: ANTIEMÉTICOS: dexametasona, metoclopramida; CIRURGIA, Ambulatorial; COMPLICAÇÕES: náusea, vômito

\section{SUMMARY}

Bedin A, Pinho MSL, Zanotelli CT, Caldart AS, Turazzi JC, Castro RAC - Dexamethasone Compared to Metoclopramide in the Prophylaxis of Emesis in Children Undergoing Ambulatory Surgical Procedures

BACKGROUND AND OBJECTIVES: Postoperative vomiting is a common and unpleasant complication. Currently, however, mathematical models, such as number necessary to treat (NNT) and relative risk reduction (RRR), have been useful in the decision of which medication to use for prophylaxis. This study aimed at verifying whether dexamethasone, as compared to metoclopramide, decreases the incidence of vomiting when intravenously administered to children anesthetized with sevoflurane for ambulatory pediatric surgeries.

METHODS: Two hundred and thirty seven male children, aged 11 months to 12 years, physical status ASA I and II, undergoing hernia repair were included in this study. They were premedicated with oral midazolam. Anesthesia was induced and maintained with sevoflurane, nitrous oxide, and $1 \mu \mathrm{g} . \mathrm{kg}^{-1}$ fentanyl. Patients were divided in two groups: group $D$ patients $(n=118)$ were given $150 \mu \mathrm{g} \cdot \mathrm{kg}^{-1}$ dexamethasone at induction while group $M(n=119)$ received $150 \mu \mathrm{g} \cdot \mathrm{kg}^{-1}$ metoclopramide at induction. The following parameters were evaluated: incidence of vomiting in the first 4 postoperative hours (PO), incidence of vomiting between 4 and 24 PO hours, NNT of both medications and RRR of dexamethasone as compared to metoclopramide.

RESULTS: The incidence of vomiting was $9.32 \%$ for group $D$ and $33.61 \%$ for group $M$ during the first $4 P O$ hours, and $1.69 \%$ with dexamethasone and $3.36 \%$ with metoclopramide between 4 and 24 PO hours. RRR of dexamethasone related to metoclopramide in the first 4 hours was $72 \%$. The number necessary to treat (NNT) for dexamethasone was 3.25 and for metoclopramide it was 15.66.

CONCLUSIONS: Dexamethasone is more effective than metoclopramide in decreasing the incidence of vomiting when used during anesthetic induction with sevoflurane associated to nitrous oxide and fentanyl.

Key Words: ANTIEMETICS: dexamethasone, metoclopramide; COMPLICATIONS: nausea, vomiting; SURGERY, Ambulatory 


\section{INTRODUÇÂO}

$A^{s}$ s náuseas e os vômitos estão entre os mais freqüentes efeitos colaterais da anestesia ${ }^{1}$. Estas complicações são causas comuns de retardo na alta após a recuperação anestésica, ansiedade, desidratação e distúrbios metabólicos em pacientes submetidos a cirurgias ambulatoriais ${ }^{2,3}$.

Embora a incidência de vômitos pós-operatórios em crianças seja maior que em adultos, a administração profilática de antieméticos é controversa ${ }^{1,2}$, alguns autores recomendam, outros afirmam que não deva ser rotineiramente utilizada porque a maioria dos agentes comumente empregados apresentam efeitos adversos como sedação, cefaléia, agitação ou sintomas extrapiramidais ${ }^{1,2}$. A utilização da metoclopramida como antiemético profilático é freqüente, porém este agente possui limitações devido à baixa eficiência e à possibilidade de ocorrência de efeitos colaterais indesejáveis tais como sintomas extrapiramidais ${ }^{2}$. A dexametasona é um corticosteróide de baixo custo, baixa incidência de efeitos colaterais, efeito antiemético prolongado em pacientes oncológicos e reduz acentuadamente a incidência de vômitos pós-operatórios (VPO) em crianças submetidas a amigdalectomias ${ }^{2}$. Entretanto, o número de estudos com a dexametasona na profilaxia de VPO ainda é pequeno, principalmente os relacionados a cirurgias pediátricas ambulatoriais tais como herniorrafias inguinais ${ }^{2-4}$.

Recentemente, alguns modelos matemáticos têm passadoa fazer parte da pesquisa clínica para tornar mais prática a difícil decisão de utilizar ou não uma medicação. Estes modelos matemáticos, tais como número necessário para tratar (NNT) e redução do risco relativo (RRR), auxiliam a tornar mais clara a eficiência de uma determinada medicação ${ }^{5-9}$ O objetivo desta pesquisa foi avaliar a hipótese de que a dexametasona é mais eficiente do que a metoclopramida na redução da incidência de vômitos pós-operatórios, quando utilizada durante a indução em anestesias com sevoflurano associado ao óxido nitroso e fentanil em crianças submetidas a procedimentos cirúrgicos ambulatoriais.

\section{MÉTODO}

Estudo clínico prospectivo, aleatório, realizado no Hospital Municipal São José, Joinville, SC, após a aprovação pelo Conselho de Ética em Pesquisa da Universidade da Região de Joinville, e o consentimento informado dos pais ou responsável legal de cada paciente. Foram incluídas crianças de 11 meses a 12 anos, estado físico ASAI ou II, do sexo masculino, com exame parasitológico de fezes negativo, sem história pregressa de vômitos pós-operatórios em cirurgias anteriores ou cinetose, que foram submetidos à herniorrafia inguinal em caráter ambulatorial, sob anestesia geral (técnica padronizada).

Para a elaboração das tabelas de alocação para distribuir os pacientes neste estudo foi utilizada a função número aleatório do programa Microsoft Excel XP ${ }^{\circledR}$. A medicação pré-anestésica constou de midazolam $\left(0,3 \mathrm{mg} \cdot \mathrm{kg}^{-1}\right)$, por via oral, uma hora antes da cirurgia. A indução foi feita com sevoflurano, fentanil $\left(1 \mu \mathrm{g} \cdot \mathrm{kg}^{-1}\right)$, oxigênio e óxido nitroso a $50 \%$; em seguida realizada a inserção de máscara laríngea ou intubação traqueal. Infiltração no local da incisão com bupivacaína (até $\left.2 \mathrm{mg} \cdot \mathrm{kg}^{-1}\right)$ e dipirona por via venosa $\left(20 \mathrm{mg} \cdot \mathrm{kg}^{-1}\right)$. A manutenção da anestesia foi feita com sevoflurano, oxigênio e óxido nitroso a 50\%. Para a hidratação foi utilizada solução glicosada a $5 \%\left(10 \mathrm{~mL} . \mathrm{kg}^{-1}\right)$ a cada hora. Após a alta foi prescrito paracetamol $\left(10 \mathrm{mg} \cdot \mathrm{kg}^{-1}\right)$ por via oral, a cada 6 horas.

Para avaliar uma medicação cuja expectativa é reduzir a incidência de vômitos em $50 \%$ em uma população submetida a uma técnica anestésico-cirúrgica em que a incidência esperada de VPO é $40 \%$, conforme levantamentos prévios na instituição, e considerando alfa $=0,05$ e beta $=0,2$ como aceitáveis, são necessários, no mínimo, 91 pacientes em cada grupo, conforme quadro publicado por Apfel ${ }^{9}$. Por este motivo, 240 pacientes foram divididos aleatoriamente em dois grupos, o grupo $D(n=120)$ recebeu $150 \mu \mathrm{g} \cdot \mathrm{kg}^{-1}$ de dexametasona por via venosa na indução; o grupo $M(n=120)$ recebeu $150 \mu \mathrm{g} . \mathrm{kg}^{-1}$ de metoclopramida por via venosa na indução. Quando ocorreram vômitos no pós-operatório foi utilizado ondansetron $\left(100 \mu \mathrm{g} \cdot \mathrm{kg}^{-1}\right)$ por via venosa em dose única. As variáveis analisadas foram ocorrência de vômitos nas primeiras quatro horas de pós-operatório (sendo vômito definido como expulsão de conteúdo gástrico), ocorrência de vômitos múltiplos nas primeiras quatro horas de pós-operatório (definidos como episódios de vômitos distintos observados em intervalos superiores a cinco minutos) e ocorrência de vômitos nas primeiras 24 horas de pós-operatório (verificados através de contato telefônico). Foram avaliados os efeitos colaterais da metoclopramida (sedação, inquietação, diarréia, agitação, depressão do sistema nervoso central), da dexametasona (epigastralgia, ansiedade, insônia) e necessidade de internação por vômito pós-operatório. O cálculo da redução do risco relativo (RRR) de VPO foi analisado através da fórmula $R R R=(1-(P R D / P R M)) \times 100$, sendo $P R D$ a probabilidade de redução de VPO com a dexametasona e PRM a probabilidade de redução com a metoclopramida durante as 24 horas, para vômitos recorrentes e entre quatro e 24 horas. O cálculo do número necessário para tratar (NNT) da dexametasona e da metoclopramida foi analisado através da fórmula NNT=1/(PEA-POT), sendo PEA a probabilidade do evento adverso esperado e POT, a probabilidade observada no grupo em tratamento durante as primeiras quatro horas de pós-operatório.

A caracterização da população estudada foi feita pela estatística descritiva (média e desvio-padrão). Foi utilizado o teste $t$ de Student para comparar as diferenças entre os valores médios das variáveis idade, massa corporal e altura, e o teste do Qui-quadrado $\left(X^{2}\right)$ para verificar se houve diferença significativa na incidência de vômitos, com um limite de confiança de $95 \%(p=0,05)$. O programa utilizado foi o Excel $X P^{\circledR}$.

\section{RESULTADOS}

Participaram do estudo 237 pacientes, sendo 118 no grupo $D$ (dexametasona) e 119 no grupo M (metoclopramida). (Tabela I). Dois pacientes no grupo $\mathrm{D}$ e um paciente no grupo $\mathrm{M}$ fo- 
ram excluídos do estudo porque não foi possível contato telefônico após a alta hospitalar. Três pacientes no grupo D e dois no grupo M necessitaram intubação traqueal, porém nenhum deles apresentou VPO dentro de 24 horas. Não houve diferença significativa com relação aos itens massa corporal, altura, idade, tempo cirúrgico e tempo entre o término da cirurgia e a alta hospitalar (tempo de recuperação pós-operatória) entre os grupos pelo teste $t$ de Student para duas amostras (Tabelas I e II).

Tabela I - Características Gerais dos Pacientes

\begin{tabular}{ccc}
\hline Variáveis & $\begin{array}{c}\text { Grupo D } \\
(\mathrm{n}=118)\end{array}$ & $\begin{array}{c}\text { Grupo M } \\
(\mathrm{n}=119)\end{array}$ \\
\hline $\begin{array}{c}\text { Idade (anos) } \\
(\mathrm{p}=0,47)^{*}\end{array}$ & $5,27 \pm 3,33$ & $5,56 \pm 2,93$ \\
$\begin{array}{c}\text { Massa }(\mathrm{kg}) \\
(\mathrm{p}=0,08)^{*}\end{array}$ & $19,2 \pm 7,94$ & $20,89 \pm 7,22$ \\
$\begin{array}{c}\text { Altura }(\mathrm{cm}) \\
(\mathrm{p}=0,63)^{*}\end{array}$ & $108,31 \pm 21,29$ & $109,57 \pm 20,14$ \\
$\begin{array}{c}\text { Estado físico } \\
\text { ASA I }\end{array}$ & 110 & 106 \\
ASA II & 8 & 13 \\
\hline
\end{tabular}

* Média \pm DP e Probabilidade $(p)$ pelo teste $t$ de Student

Tabela II - Tempo Cirúrgico e Tempo de Recuperação Pós-Operatória

\begin{tabular}{lcc}
\hline & $\begin{array}{c}\text { Grupo D } \\
(\mathrm{n}=118)\end{array}$ & $\begin{array}{c}\text { Grupo M } \\
(\mathrm{n}=119)\end{array}$ \\
\hline $\begin{array}{l}\text { Tempo cirúrgico (min) } \\
(\mathrm{p}=0,07)\end{array}$ & $29,11 \pm 7,96$ & $191,87 \pm 40,17$ \\
$\begin{array}{l}\text { Recuperação (min) } \\
(\mathrm{p}=0,63)\end{array}$ & $31,17 \pm 9,55$ & $194,51 \pm 45,01$ \\
\hline
\end{tabular}

Média \pm DP e Probabilidade $(p)$ pelo teste $t$ de Student

A incidência de vômitos de 9,32\% (11) no Grupo D e 33,61\% (40) no grupo M durante as primeiras quatro horas após o ato anestésico-cirúrgico apresentou diferença estatisticamente significativa entre os grupos, $X^{2}=5,36$, para um nível de sig-

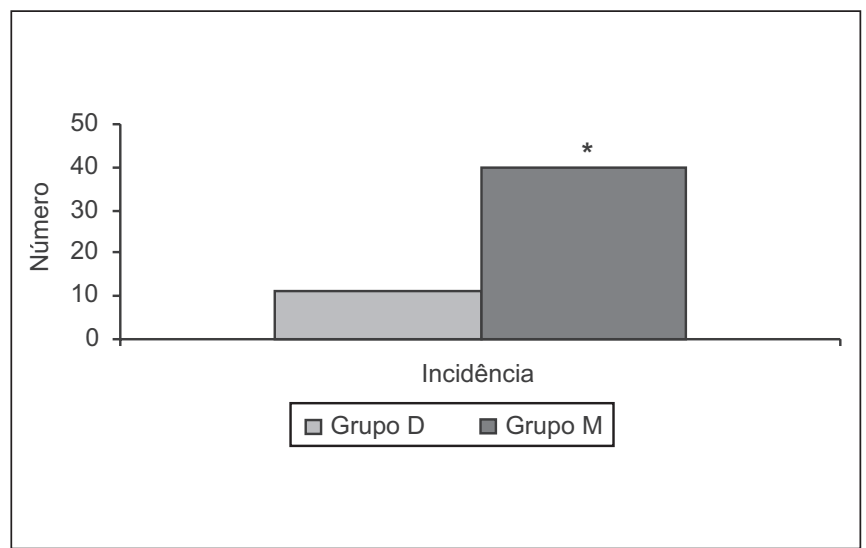

Figura 1 - Incidência de Vômitos nas Primeiras Quatro Horas de Pós-Operatório. Um episódio

* Diferença estatística significativa $(p<0,05)$

Dados expressos em números absolutos nificância de 0,05, 1 grau de liberdade e um Qui-quadrado crítico de 3,84 (Figura 1).

A incidência de vômitos recorrentes (mais de 1 episódio) de 5,08\% (6) no Grupo D e 15,97\%(19), no grupo M, durante as primeiras quatro horas após o ato anestésico-cirúrgico, não apresentou diferença estatística significativa entre os grupos, $X^{2}=0,002$, para um nível de significância de $0,05,1$ grau de liberdade e um Qui-quadrado crítico de 3,84 (Figura 2).

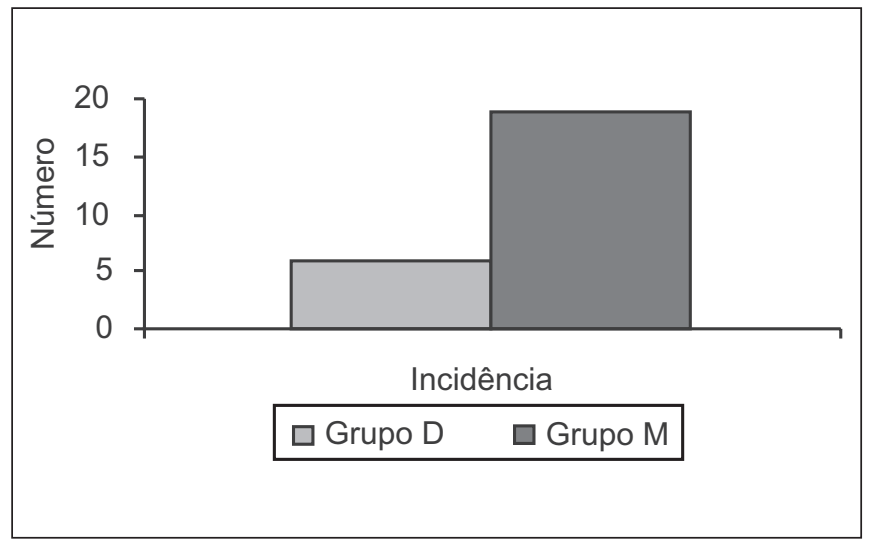

Figura 2 - Incidência de Vômitos nas Primeiras Quatro Horas de Pós-Operatório. Multiplos episódios

Diferença estatística não significativa entre os grupos $(\mathrm{p}>0,05)$

Dados expressos em números absolutos

A incidência de vômitos de 1,69\% (2) no Grupo D e de 3,36\% (4), no grupo M, após a alta hospitalar e antes de completar as primeiras vinte e quatro horas após o ato anestésico-cirúrgi$\mathrm{co}$, não apresentou diferença estatística significativa, $\mathrm{X}^{2}=$ 0,41 , para um nível de significância de $0,05,1$ grau de liberdade e Qui-quadrado crítico de 3,84 (Figura 3). Todos os pacientes que apresentaram vômitos após a alta estavam entre aqueles que já haviam apresentado vômitos nas primeiras quatro horas de pós-operatório.

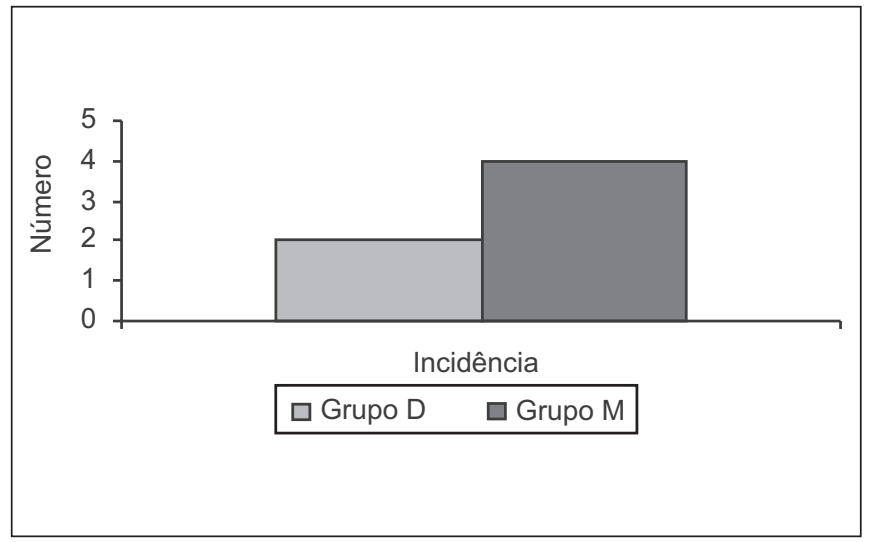

Figura 3 - Incidência de Vômitos após a Alta Hospitalar Diferença estatística não significativa entre os grupos $(p>0,05)$

Dados expressos em números absolutos 
Não foram observados efeitos colaterais com a dexametasona nem com a metoclopramida. Não houve necessidade de internação por VPO.

A redução do risco relativo ( $R R R$ ) da dexametasona em relação a metoclopramida foi $72 \%$ nas primeiras quatro horas, $72,27 \%$ relativos a vômitos recorrentes nas primeiras quatro horas e $50 \%$ entre quatro e 24 horas (Figura 4 ).

O número necessário para tratar (NNT) da dexametasona foi 3,25 e o da metoclopramida foi 15,66 (Figura 5).

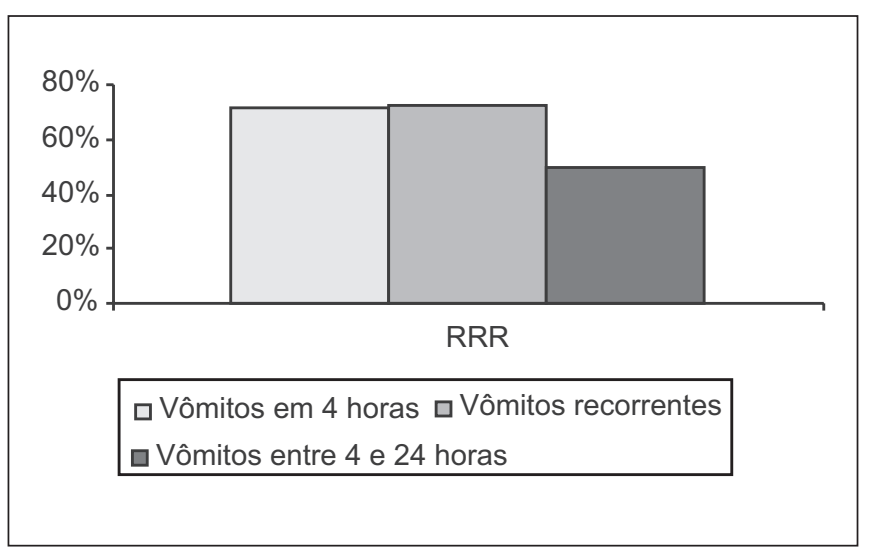

Figura 4 - Redução do Risco Relativo (RRR) da Dexametasona em Relação à Metoclopramida

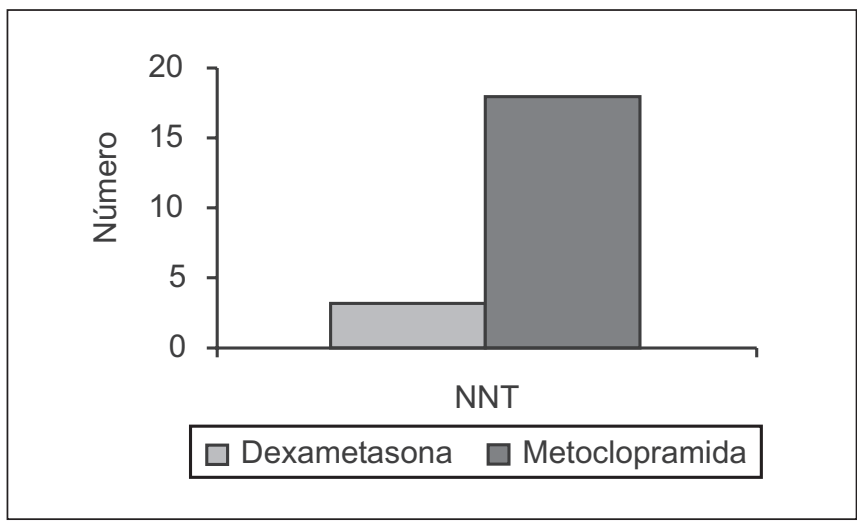

Figura 5 - Número Necessário para Tratar (NNT) da Dexametasona e da Metoclopramida

\section{DISCUSSÃo}

A náusea é uma sensação subjetiva e desagradável na região do epigástrio e orofaringe, associada a uma urgente necessidade de vomitar ${ }^{3}$. O vômito é caracterizado por contração espasmódica do diafragma, parede muscular abdominal, músculos respiratórios e parede torácica, seguido pela expulsão de conteúdo gástrico através da boca como resultado dessa contratura da parede abdominal, diafragma e abertura do cárdia ${ }^{10}$. A náusea é um fenômeno difícil para a criança descrever e por este motivo a maioria dos estudos com populações pediátricas tem considerado apenas vômitos. Isto explica algumas das diferenças encontradas nos re- sultados de estudos feitos com crianças e adultos ${ }^{4,11-13}$. O centro do vômito, localizado na formação reticular do tronco encefálico, controla e coordena o complexo processo do vômito. Esta área recebe impulsos de outras regiões dentro do sistema nervoso central, incluindo a área quimiorreceptora do gatilho, cerebelo, sistema vestibular, centros corticais e do núcleo do trato solitário ${ }^{3}$. Estas áreas são ricas em receptores serotoninérgicos, muscarínicos, histamínicos, opióides e dopaminérgicos. O bloqueio destes receptores tem sido postulado como mecanismo de ação das drogas antieméticas. O impulso eferente do centro do vômito segue via nervo frênico e nervos espinhais da musculatura da parede abdominal durante o ato do vômito ${ }^{3,13,14}$.

Foi relatado um índice geral de VPO de 33,2\% em 983 pacientes pediátricos submetidos a diversas cirurgias em um hospital comunitário ${ }^{12}$. Outro estudo relatou que a incidência de VPO pode variar de $15 \%$ a $54 \%$ em cirurgias de abdômen inferior tais como herniorrafias, hidrocelectomias e orquidopexias ${ }^{14}$. Um grande número de fatores contribui para a incidência de VPO, e nem todos podem ser controlados pelo anestesiologista. Pacientes pediátricos apresentam incidência maior de VPO que a população adulta ${ }^{2,12}$. Durante a infância a incidência de VPO é igual entre os sexos femininoe masculino, mas após a adolescência, a incidência de VPO é maior entre as mulheres. Tanto crianças como adultos com história de cinetose ou VPO prévios apresentam uma possibilidade maior de VPO. Pacientes que recebem medicação pré-anestésica e estão mais calmos no início do procedimento cirúrgico apresentam menor incidência de VPO ${ }^{2}$. Estudos anteriores haviam sugerido que pacientes obesos apresentavam maior possibilidade de VPO ${ }^{2}$. Entretanto, dados atuais não demonstram relação entre obesidade e incidência aumentada de VPO ${ }^{3}$. Pacientes fumantes adultos apresentam menor incidência de VPO, mas não existem dados disponíveis sobre a incidência de VPO em crianças que convivem com adultos fumantes ${ }^{2}$. Certos procedimentos cirúrgicos estão associados com alta incidência de VPO, como cirurgia de estrabismo, ouvido, nariz e garganta, operações sobre o ouvido médio, amigdalectomias, orquidopexias e cirurgias craniofaciais. A incidência de VPO aumenta com a duração da cirurgia em torno de $6 \%$ a cada 30 minutos. Opióides, óxido nitroso, anestésicos voláteis e anticolinesterásicos aumentam a incidência de VPO. Entretanto certos anestésicos venosos, como o propofol, diminuem a incidência de VPO. Estudos têm demonstrado que existe uma correlação entre a intensidade da dor e a incidência de VPO em crianças ${ }^{2}$. Na sala de recuperação movimentos bruscos podem desencadear VPO. O horário da primeira ingesta, após a intervenção cirúrgica, pode influenciar a incidência de VPO durante o período de recuperação ${ }^{2}$. A probabilidade de VPO em adultos pode ser estimada pelo número de fatores de risco associados como sexo feminino, história prévia de VPO ou cinetose, não fumante e opióides na analgesia pós-operatória. Se nenhum, um, dois, três ou quatro fatores estão presentes, o risco de VPO é aproximadamente 10\%, 20\%, 40\%, $60 \%$ e $80 \%$, respectivamente ${ }^{9}$. Entretanto, em crianças, os fatores preditivos apresentam somente uma vaga correlação com as inci- 
dências reais observadas ${ }^{12}$. Existem dados suficientes para sugerir que a profilaxia do vômito em pacientes com moderado (30\% a $60 \%$ ) ou elevado risco (acima de $60 \%$ ) é justificada, porque reduz custos e melhora a satisfação dos pacientes. Entretanto não seria justificado em pacientes de baixo risco (menos que 30\%) ${ }^{1,9,14}$.

Muitas medicações têm sido utilizadas na profilaxia de VPO, após sua eficácia ter sido estabelecida em estudos clínicos ${ }^{9}$. A escolha da medicação deve ser baseada na evidência de sua eficiência, perfil de baixos efeitos colaterais e baixo custo ${ }^{1,9}$.

O mecanismo básico de ação da metoclopramida é facilitar a liberação da acetilcolina dos neurônios entéricos. Esta ação pode ser mediada por uma variedade de mecanismos. A supressão de interneurônios inibitórios pelo antagonismo dos receptores $5-\mathrm{HT}_{3}$ e a estimulação dos neurônios excitatórios por receptores $5-\mathrm{HT}_{4}$ podem ocorrer. Ametoclopramida é antagonista da dopamina $\left(D_{2}\right)$. É muito provável que os efeitos dopaminérgicos centrais contribuam para os efeitos antieméticos e inibidores de náuseas da metoclopramida ${ }^{2}$. A metoclopramida em doses elevadas (1 a $2 \mathrm{mg} \cdot \mathrm{kg}^{-1}$ ) foi usada com sucesso para prevenir ou reduzir o vômito induzido por quimioterapia (VIQ). Em doses bem mais baixas $(0,1$ a 0,2 $\left.\mathrm{mg} . \mathrm{kg}^{-1}\right)$ foi utilizada para tratar VPO ${ }^{2}$. Estas doses foram utilizadas em uma tentativa de diminuir os efeitos sedativos e a incidência elevada das reações distônicas associadas com as doses empregadas em VIQ. Mesmo quando utilizada em doses baixas, as crianças parecem mais predispostas a estes efeitos colaterais do que os adultos. A eficiência relatada da metoclopramida, quando administrada nas doses mais baixas usadas para VPO, é muito variável ${ }^{2}$. Um grande número de fatores pode contribuir para resultados inconsistentes, incluindo a via de administração, tipo de procedimento cirúrgico e diferenças na técnica anestésica. Em razão da meia-vida curta da metoclopramida, é improvável que a administração durante a indução da anestesia possa produzir algum efeito antiemético após o período de emergência ${ }^{2,3}$. Revisões sistemáticas têm questionado a eficiência da metoclopramida na dose de $10 \mathrm{mg} \mathrm{em}$ adultos ${ }^{4}$. Uma revisão sistemática afirmou que para uma dose de $250 \mu \mathrm{g} . \mathrm{kg}^{-1}$ o NNT para a metoclopramida em crianças é $7,9^{15}$.

Os corticosteróides, particularmente a dexametasona, foram usados no VIQ por muitos anos. Porém, o mecanismo exato ou mecanismos de ação dos corticosteróides na prevenção da náusea ou na profilaxia do vômito é desconhecido; sugere-se o antagonismo de prostaglandinas, a liberação de endorfinas, e a depleção do triptofano. Entretanto, não se conhece se estes efeitos são periféricos ou mediados centralmente ${ }^{2}$. É também possível que os efeitos antiinflamatórios ou estabilizadores de membrana contribuam para os efeitos antieméticos destas drogas ${ }^{2}$. Adexametasona demonstrou diminuir a incidência de VPO, quando administrada profilaticamente. As doses mais freqüentemente estudadas nos adultos foram de $8 \mathrm{mg}$ a $10 \mathrm{mg}$ por via venosa. Nas crianças as doses típicas variaram de $0,1 \mathrm{mg} \cdot \mathrm{kg}^{-1}$ a 1,5 $\mathrm{mg} \cdot \mathrm{kg}^{-1}$. Nestas doses, o NNT para prevenir VPO foram 7,1 nos adultos e 3,8 em crianças ${ }^{16}$. Entretanto, é provável que doses menores que $5 \mathrm{mg}$ em adultos também sejam efetivas

${ }^{2}$. A administração de $10 \mathrm{mg}$ de dexametasona venosa antes da indução da anestesia foi mais eficaz do que $10 \mathrm{mg}$ administrados no fim da cirurgia para evitar VPO durante as primeiras duas horas de pós-operatório ${ }^{1,2}$

O ideal, em termos estatísticos, é comparar a medicação antiemética com um grupo controle em que é utilizado placebo na profilaxia de vômitos pós-operatórios. Porém, não é justificável, por razões éticas, a negação de profilaxia e tratamento, quando um estudo de metanálise já tenha quantificado anteriormente o efeito de um antiemético, sendo aconseIhável comparar o novo antiemético a um antiemético com evidência já estabelecida ${ }^{9}$. A metoclopramida é um agente antiemético utilizado há mais de quarenta anos ${ }^{4}$, apesar de sua eficiência ser questionada atualmente ${ }^{1,4,15}$. Adexametasona é um agente utilizado como antiemético em pacientes oncológicos há mais de dez anos, porém o número de estudos realizados na profilaxia de vômito em pós-operatório em crianças ainda é pequeno ${ }^{1,2,9,16}$.

Modelos matemáticos tais como redução do risco relativo e número necessário para tratar são exemplos de como a matemática pode ser útil na tomada de decisões em Anestesiologia. A redução de risco relativo (RRR) é calculada pela fórmula RRR =(1-(PRB/PRA) $) \times 100$, sendo PRB a probabilidade de redução de VPO com a droga B e PRA, a probabilidade de redução com a droga $A$. A redução do risco relativo é expressa em porcentagem ${ }^{6,7}$. No presente estudo o índice RRR= $72 \%$, quando se compara a dexametasona à metoclopramida, durante as primeiras quatro horas de pós-operatório, expressa que a dexametasona é $72 \%$ mais eficiente que a metoclopramida. O número necessário para tratar (NNT) é obtido através da fórmula NNT=1/(PEA-POT), sendo PEA a probabilidade do evento adverso esperado e POT, a probabilidade observada no grupo em tratamento ${ }^{6,7}$. A dexametasona, neste estudo durante as quatro primeiras horas de pós-operatório, demonstrou um NNT $=3,25$, superior à metoclopramida NNT = 15,66, isto quer dizer que com a dexametasona é necessário que sejam tratados 3,25 pacientes para que se evite um episódio de VPO, enquanto com a metoclopramida é necessário tratar 15,66 para se evitar um episódio de VPO. A compreensão dos trabalhos sobre VPO melhorou quando foi estendido o uso dos conceitos de redução de risco relativo e de número necessário para tratar a este campo de estudo. Esta aproximação determinou o número dos pacientes que necessitam ser tratados para que um paciente obtenha o efeito desejado. A vantagem desta aproximação é permitir comparações indiretas de vários regimes terapêuticos. Estes modelos matemáticos não se contrapõem aos tradicionais testes estatísticos, mas acrescentam ${ }^{6-8}$.

É freqüente a citação na literatura de que o VPO seja um empecilho para a alta precoce, o que é justamente contrário aos objetivos da anestesia ambulatorial ${ }^{1,10}$. Porém, não houve diferença estatística significativa entre os dois grupos, aqui estudados, com relação ao tempo entre o término da cirurgia e a alta hospitalar e não houve necessidade de internações devido a vômito incoercível. 
Este estudo concluiu que a dexametasona demonstrou ser mais eficiente que a metoclopramida na redução da incidência de vômitos pós-operatórios, quando utilizada durante a indução em anestesias com sevoflurano associado ao óxido nitroso e fentanil, em crianças do sexo masculino submetidas a herniorrafias inguinais em regime ambulatorial.

\section{Dexamethasone Compared to Metoclopra- mida in the Prophylaxis of Emesis in Children Undergoing Ambulatory Surgical Procedures}

Antonio Bedin, TSA, M.D.; Mauro de Souza Leite Pinho; Cladir Terezinha Zanotelli; Ângela Sílvia Caldart; Jurandir Coan Turazzi, TSA, M.D.; Renato Almeida Couto de Castro, TSA, M.D.

\section{INTRODUCTION}

Nausea and vomiting are among the most frequent anesthetic side-effects ${ }^{1}$. These complications are common causes of late discharge after anesthetic recovery, anxiety, dehydration and metabolic changes in patients submitted to ambulatory surgeries ${ }^{2,3}$.

Although the incidence of postoperative vomiting is higher in children as compared to adults, prophylactic antiemetics are controversial ${ }^{1,2}$; some authors recommend them while others state that they should not be routinely used because most popular agents present adverse effects, including sedation, headache, agitation or extrapyramidal symptoms ${ }^{1,2}$.

Metoclopramide is commonly used as prophylactic antiemetic drug. However, its use is limited by low efficiency and the possibility of undesirable side effects, such as extrapyramidal symptoms ${ }^{2}$. Dexamethasone is a low cost steroid, with low incidence of side effects, prolonged antiemetic effect in oncologic patients, and markedly decreases the incidence of postoperative vomiting in children submitted to tonsillectomy ${ }^{2}$. However, there are still few studies with dexamethasone to prevent POV, especially POV related to ambulatory pediatric surgeries such as inguinal hernia correction ${ }^{2-4}$.

Recently, some mathematical models have become part of clinical research to aid in the difficult decision of adopting or not a drug. These mathematical models, such as number necessary to treat (NNT) and relative risk reduction (RRR) help clarifying the efficacy of a given medication ${ }^{5-9}$.

The purpose of this study was to evaluate whether dexamethasone is more effective than metoclopramide in decreasing postoperative vomiting when used during anesthetic induction with sevoflurane, nitrous oxide and fentanyl, in children submitted to ambulatory surgical procedures.

\section{METHODS}

This clinical prospective study was carried out at Hospital Municipal São José, Joinville, SC, after the Regional Research Ethics Committee approval and the informed consent of children's parents or guardians. Inclusion criteria were male children aged 11 months to 12 years, physical status ASA I or II, with negative feces exam and no history of postoperative vomiting in previous surgeries or motion sickness, submitted to ambulatory inguinal hernia correction under general anesthesia (standard technique).

To prepare allocation tables and distribute patients in this study, Microsoft Excel $\mathrm{XP}^{\circledR}$ random number function was used. Patients were premedicated with $0.3 \mathrm{mg} \cdot \mathrm{kg}^{-1}$ oral midazolam one hour before surgery. Anesthesia was induced with sevoflurane, $1 \mu \mathrm{g} \cdot \mathrm{kg}^{-1}$ fentanyl and $50 \%$ oxygen and nitrous oxide, followed by laryngeal mask or tracheal intubation. Incision site was infiltrated with up to $2 \mathrm{mg} \cdot \mathrm{kg}^{-1}$ bupivacaine and $20 \mathrm{mg} \cdot \mathrm{kg}^{-1}$ dipirone was intravenously administered. Anesthesia was maintained with sevoflurane and $50 \%$ oxygen and nitrous oxide. Hydration was achieved with $5 \%$ glucose solution ( $\left.10 \mathrm{~mL} . \mathrm{kg}^{-1}\right)$ every hour. Oral paracetamol (10 mg. $\left.\mathrm{kg}^{-1}\right)$ every 6 hours was prescribed at hospital discharge.

To evaluate a drug expected to decrease vomiting in $50 \%$ of a population submitted to surgical procedures in which the expected POV incidence is $40 \%$ (according to our previous surveys) and considering acceptable alpha $=0.05$ and beta $=$ 0.2 , at least 91 patients are needed in each group, according to chart published by Apfel ${ }^{9}$. For this reason, 240 patients were randomly divided in two groups: group $D(n=120)$ received $150 \mu \mathrm{g} . \mathrm{kg}^{-1}$ intravenous dexamethasone and group $\mathrm{M}$ $(n=120)$ received $150 \mu \mathrm{g} . \mathrm{kg}^{-1}$ intravenous metoclopramide, both at induction. Bolus intravenous ondansetron (100 $\mu \mathrm{g} . \mathrm{kg}^{-1}$ ) was used to treat postoperative vomiting.

Evaluated variables were the incidence of vomiting in the first 4 postoperative hours (vomiting defined as gastric content expelling), the incidence of multiple vomiting episodes in the first 4 postoperative hours (defined as different vomiting episodes observed in intervals higher than 5 minutes) and vomiting in the first 24 postoperative hours (confirmed by telephone contact).

The following parameters were also evaluated: metoclopramide side effects (sedation, agitation, diarrhea, central nervous system depression), dexamethasone side effects (epigastric pain, anxiety, insomnia) and change to inpatient regimen due to postoperative vomiting. The relative risk reduction $(R R R)$ of $P O V$ was calculated with the formula $R R R=(1-(P R D / P R M)) \times 100$, where PRD is the probability of POV decrease with dexamethasone and PRM the probability of POV decrease with metoclopramide during 24 hours for recurrent vomiting and between 4 and 24 hours. The number necessary to treat (NNT) for dexamethasone and metoclopramide was calculated by the formula NNT=1/(PEA-POT), where PEAis the expected probability of 
the adverse event and POT the observed probability of the event in the group treated during the first 4 postoperative hours.

Descriptive statistics (mean and standard deviation) was used to characterize studied population. Student's $t$ test was used to compare differences between mean values of age, body mass index and height, and Chi-square test $\left(\mathrm{X}^{2}\right)$ was used to check whether there has been significant difference in the incidence of vomiting between groups, with a confidence limit of $95 \%(p \leq 0.05)$. Excel XP ${ }^{\circledR}$ was our chosen computer program.

\section{RESULTS}

Two hundred and thirty seven patients, 118 in group D (dexamethasone) and 119 in group M (metoclopramide) took part in this study (Table I). Two group D patients and one group $M$ patient were excluded because telephone contact was impossible after hospital discharge. Three group $D$ patients and 2 group M patients needed tracheal intubation, but none of them presented POV within 24 hours. There were no significant differences in body mass index, height, age, surgery duration and time between surgery completion and hospital discharge (postoperative recovery period) according to Student's $t$ test for two samples (Tables I and II).

Table I - Demographics Data

\begin{tabular}{lcc}
\hline Variables & $\begin{array}{c}\text { Group D } \\
(\mathrm{n}=118)\end{array}$ & $\begin{array}{c}\text { Group M } \\
(\mathrm{n}=119)\end{array}$ \\
\hline $\begin{array}{c}\text { Age (years) } \\
(\mathrm{p}=0.47)^{*}\end{array}$ & $5.27 \pm 3.33$ & $5.56 \pm 2.93$ \\
$\begin{array}{c}\text { Body mass index }(\mathrm{kg}) \\
(\mathrm{p}=0.08)^{*}\end{array}$ & $19.2 \pm 7.94$ & $20.89 \pm 7.22$ \\
$\begin{array}{c}\text { Height }(\mathrm{cm}) \\
(\mathrm{p}=0.63)^{*}\end{array}$ & $108.31 \pm 21.29$ & $109.57 \pm 20.14$ \\
$\begin{array}{c}\text { Physical status } \\
\text { ASA I }\end{array}$ & 110 & 106 \\
ASA II & 8 & 13 \\
\hline
\end{tabular}

* Mean \pm SD and Probability $(p)$ according to Student's $t$ test

Table II - Surgery and Postoperative Recovery Length

\begin{tabular}{lcc}
\hline & $\begin{array}{c}\text { Group D } \\
(\mathrm{n}=118)\end{array}$ & $\begin{array}{c}\text { Group M } \\
(\mathrm{n}=119)\end{array}$ \\
\hline $\begin{array}{l}\text { Surgery length (min) } \\
(\mathrm{p}=0.07)\end{array}$ & $29.11 \pm 7.96$ & $191.87 \pm 40.17$ \\
$\begin{array}{l}\text { Recovery (min) } \\
(\mathrm{p}=0.63)\end{array}$ & $31.17 \pm 9.55$ & $194.51 \pm 45.01$ \\
\hline
\end{tabular}

Mean \pm SD and Probability $(p)$ according to Student's t test

The incidence of vomiting of $9.32 \%(11)$ in Group D and of $33.61 \%(40)$ in group $M$ during the first 4 postoperative hours was statistically different between groups, $X^{2}=5.6$, for a significance level of $0.05,1$ level of freedom and a critical Chi-square of 3.84 (Figure 1).

The incidence of recurrent vomiting (more than 1 episode) of $5.08 \%(6)$ in group D and of $15.97 \%$ (19) in group M during the first 4 postoperative hours was not statistically different between groups, $X^{2}=0.002$, for a level of significance of $0.05,1$ level of freedom and critical Chi-square of 3.84 (Figure 2). The incidence of vomiting of $1.69 \%$ (2) in group $D$ and of $3.36 \%$ (4) in group M after hospital discharge and before completing 24 postoperative hours was not statistically different, $X^{2}=$ 0.41 , for a significance level of $0.05,1$ level of freedom and critical Chi-square of 3.84 (Figure 3 ). All patients presenting vomiting after discharge were among those who had already presented vomiting in the first 4 postoperative hours.

There were no side effects both with dexamethasone and metoclopramide. There were also no hospital admissions due to POV.

The relative risk reduction ( $R R R$ ) of dexamethasone related to metoclopramide was $72 \%$ in the first 4 hours, $72.77 \%$ for recurrent vomiting in the first 4 hours and $50 \%$ between 4 and 24 hours (Figure 4).

The number necessary to treat (NNT) was 3.25 for dexamethasone and 15.66 for metoclopramide (Figure 5).

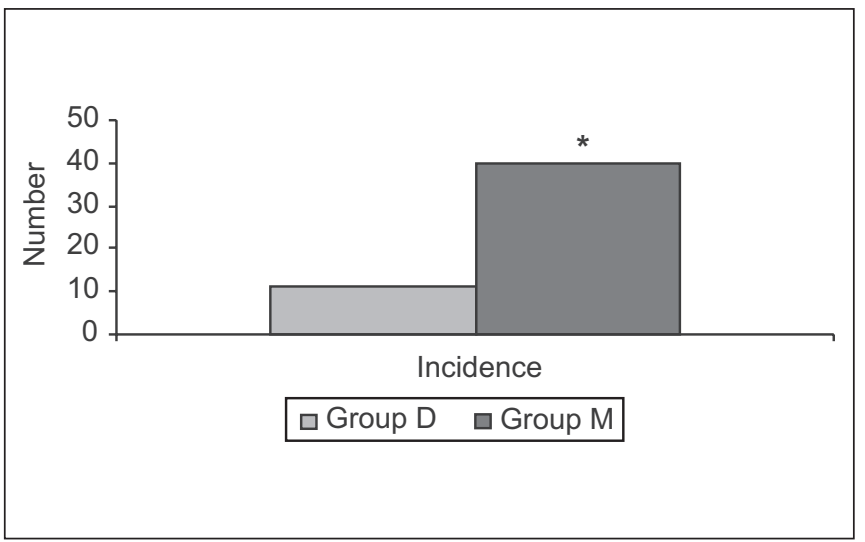

Figure 1 - Incidence of Vomiting in the First Four Postoperative Hours. One episode

* Statistically significant difference $(p<0.05)$

Data in absolute number

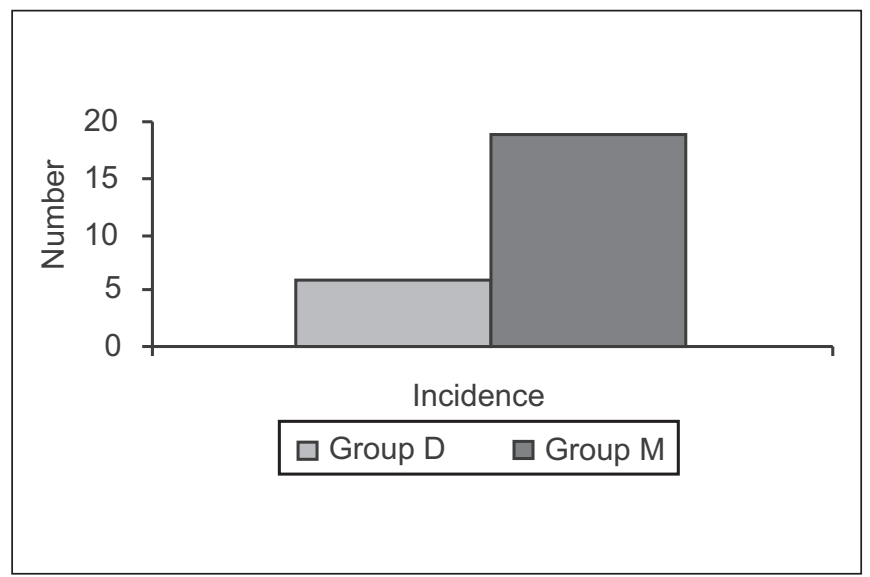

Figure 2 - Incidence of Vomiting in the First Four Postoperative Hours. Multiple episodes

No statistically significant difference $(p>0.05)$

Data in absolute number 


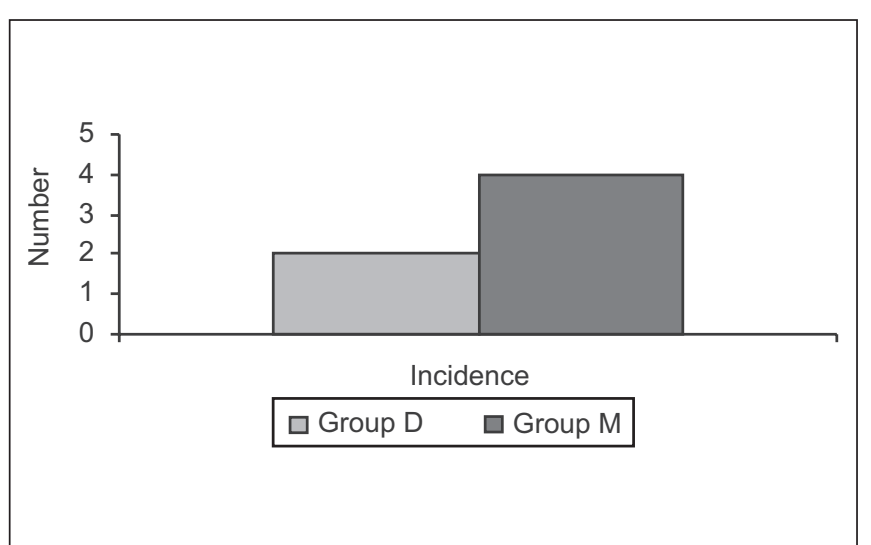

Figure 3 - Incidence of Vomiting after Hospital Discharge No statistically significant difference $(p>0.05)$ Data in absolute number

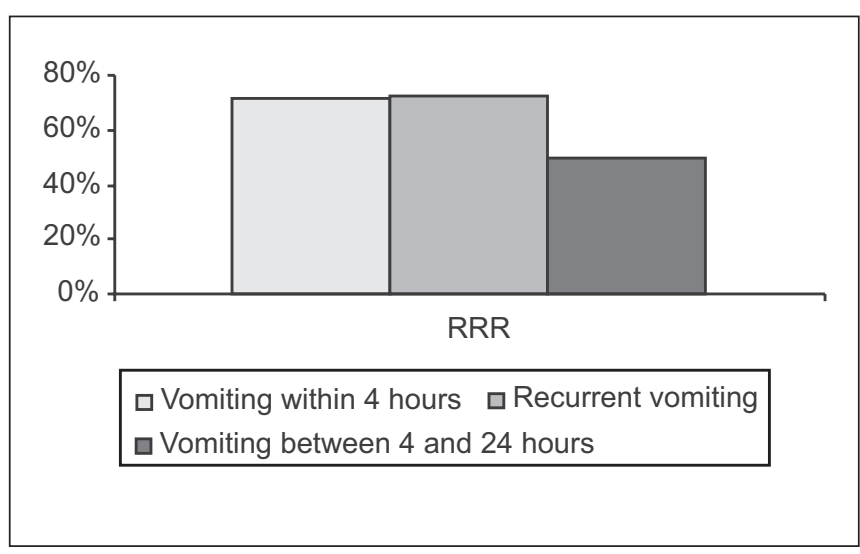

Figure 4 - Relative Risk Reduction (RRR) of Dexamethasone as compared to Metoclopramide

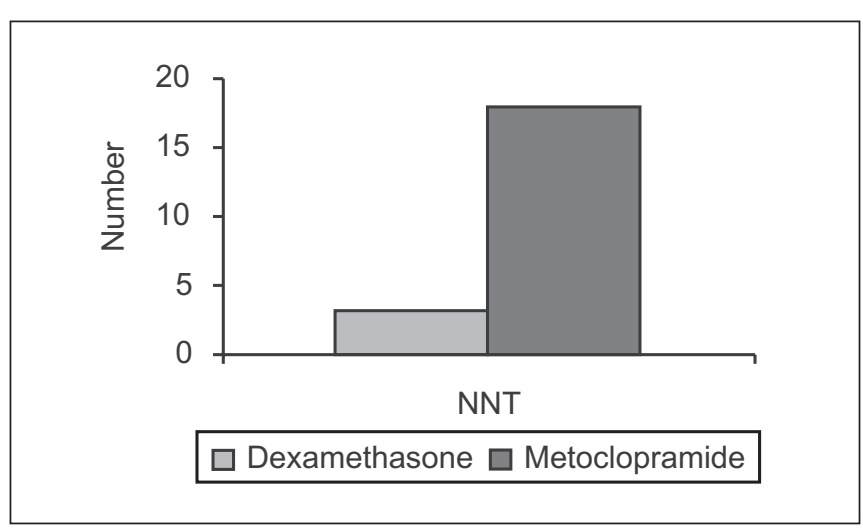

Figure 5 - Number Necessary to Treat (NNT) for Dexamethasone and Metoclopramide

\section{DISCUSSION}

Nausea is a subjective and unpleasant sensation at the epigastrium and oropharynx, associated to an urgent need to vomit ${ }^{3}$. Vomiting is characterized by diaphragm, abdominal muscles, respiratory muscles and chest wall spasmodic con- traction followed by gastric content expelling through the mouth as result of this abdominal wall and diaphragm contraction and cardia opening ${ }^{10}$.

Nausea is a difficult sensation for children to describe, so most pediatric studies have considered vomiting alone. This explains some differences in results of studies with children or adults ${ }^{4,11-13}$

Vomiting center, located in brainstem reticular formation, controls and coordinates the complex vomiting process. This area receives impulses from other central nervous system regions, including trigger chemoreceptor area, cerebellum, vestibular system, cortical centers and solitaire tract nucleus ${ }^{3}$. These areas are rich in serotoninergic, muscarinic, histaminic, opioid and dopaminergic receptors. Blocking of these receptors has been postulated as the action mechanism of antiemetic drugs. The efferent impulse of the vomiting center follows via phrenic nerve and spinal nerves of abdominal wall muscles during vomiting ${ }^{3,13,14}$.

It has been reported a general POV incidence of $33.2 \%$ in 983 pediatric patients submitted to different surgeries in a community hospital ${ }^{18}$. A different study has reported that POV may vary from $15 \%$ to $54 \%$ in lower abdomen surgeries, such as hernia correction, hydrocelectomies and orchiopexis ${ }^{14}$. A great number of factors contribute for the incidence of POV and not all can be controlled by the anesthesiologist. Pediatric patients present a higher incidence of $P O V$ as compared to adults ${ }^{2,12}$

During childhood, the incidence of POV is similar for males and females, but at adolescence, the incidence is higher among females. Both children and adults with history of motion sickness or previous POV are at higher risk of POV. Premedicated patients, who are more relaxed at the beginning of surgery, have a lower incidence of $\mathrm{POV}^{2}$.

Previous studies have suggested that obese patients were at higher risk of $\mathrm{POV}^{2}$. However, current data do not relate obesity to increased incidence of POV ${ }^{3}$. Adult smokers have a lower incidence of POV, but there are no data on the incidence of POV in children living with smoking adults ${ }^{2}$. Some surgical procedures are associated to high incidence of POV, such as strabismus, ear, nose and throat procedures, middle ear surgeries, tonsillectomies, orchiopexis and craniofacial surgeries.

The incidence of POV increases with surgery length in approximately $6 \%$ for every 30 minutes. Opioids, nitrous oxide, volatile anesthetics and anti-cholinesterase drugs increase the incidence of POV, while propofol seems to decrease it. Studies have shown correlation between pain intensity and incidence of POV in children ${ }^{2}$. Sudden movements in the recovery unit may trigger POV. Time for first postoperative meal may also influence the incidence of POV during recovery ${ }^{2}$.

The probability of POV in adults may be estimated by the number of associated risk factors, such as female gender, previous history of POV or motion sickness, non-smoker and administration of opioids for postoperative analgesia. If none, one, two, three or four factors are present, the risk of POV is approximately $10 \%, 20 \%, 40 \%, 60 \%$ and $80 \%$, respectively ${ }^{9}$. Vol. 55, № 4 , Julho - Agosto, 2005 
In children, however, predictive factors have only a weak correlation with the actual incidences ${ }^{12}$. There are enough data to suggest that vomiting prophylaxis in patients with moderate $(30 \%$ to $60 \%$ ) or high risk (above $60 \%$ ) is justified because it decreases costs and increases patients' satisfaction. However, this would not be justified in low risk patients (below $30 \%)^{1,9,14}$

Several drugs have been used to prevent POV after their efficacy has been established by clinical trials ${ }^{9}$. The choice of the drug should be based on the evidence of efficacy, few side effects and low cost ${ }^{1,9}$

Basic action of metoclopramide is facilitating acetylcholine release from enteric neurons. This action may be mediated by several mechanisms. There might be suppression of inhibitory interneurons by antagonism of $5-\mathrm{HT}_{3}$ receptors and stimulation of excitatory neurons by $5-\mathrm{HT}_{4}$ receptors. Metoclopramide is dopamine $\left(D_{2}\right)$ antagonist. It is very likely that central dopaminergic effects contribute to antiemetic and nausea inhibitory effects of metoclopramide ${ }^{2}$.

High metoclopramide doses ( 1 to $2 \mathrm{mg} \cdot \mathrm{kg}^{-1}$ ) have been successfully used to prevent or decrease chemotherapy-induced vomiting (CIV). In much lower doses (0.1 to 0.2 $\left.\mathrm{mg} \cdot \mathrm{kg}^{-1}\right)$ it has been used to treat POV ${ }^{2}$. These lower doses were used in an attempt to decrease sedative effects and the high incidence of dystonic reactions associated to CIV doses. Even with them, children seem to be more prone to these side effects as compared to adults. Reported metoclopramide efficacy, when administered in lower doses for POV, is very variable ${ }^{2}$. Agreat number of factors may contribute to inconsistent results, including administration route, type of surgery and differences in anesthetic techniques. Due to metoclopramide short half-life, it is unlikely that its administration during anesthetic induction may produce any antiemetic effect after the emergence period ${ }^{2,3}$. Systematic reviews have questioned the efficacy of $10 \mathrm{mg}$ metoclopramide in adults ${ }^{4}$.

One review has stated that for $250 \mu \mathrm{g} \cdot \mathrm{kg}^{-1}$, NNT for metoclopramide in children is $7.9^{15}$.

Steroids, especially dexamethasone, have been used for CIV for many years. However, their exact action mechanism in preventing nausea or vomiting is still unknown. Prostaglandins antagonism, endorphins release and tryptophan depletion are suggested. However it is still unknown whether these are peripheral or centrally mediated effects ${ }^{2}$.

It is also possible that anti-inflammatory or membrane stabilizing effects contribute for the antiemetic effects of these drugs $^{2}$. Prophylactic dexamethasone has decreased the incidence of POV. Most frequently studied doses in adults were intravenous $8 \mathrm{mg}$ to $10 \mathrm{mg}$. In children, typical doses vary from $0.1 \mathrm{mg} \cdot \mathrm{kg}^{-1}$ to $1.5 \mathrm{mg} \cdot \mathrm{kg}^{-1}$. In such doses, NNT to prevent POV was 7.1 for adults and 3.8 for children ${ }^{16}$. However, it is possible that doses below $5 \mathrm{mg}$ in adults are also effective ${ }^{2}$. Intravenous $10 \mathrm{mg}$ dexamethasone before anesthetic induc- tion was more effective than $10 \mathrm{mg}$ at surgery completion in preventing POV in the first 2 postoperative hours ${ }^{1,2}$.

From a strictly statistic point of view, one should compare the antiemetic drug to a control group using placebo to prevent postoperative vomiting. But it is ethically not justifiable to deny prophylaxis and treatment when a previous meta analysis study has already quantified the effect of an antiemetic drug. So, it is recommended to compare the new drug to a well-established antiemetic agent ${ }^{9}$. Metoclopramide is an antiemetic agent used for more than 40 years ${ }^{4}$ although its effectiveness is being currently questioned ${ }^{1,4,15}$. Dexamethasone is used as antiemetic agent in oncologic patients for more than 10 years, however there are still few studies on postoperative vomiting prophylaxis in children ${ }^{1,2,9,16}$.

Mathematical models, such as relative risk reduction and number necessary to treat, are examples of how mathematics may be useful for decision making in Anesthesiology. The relative risk reduction $(R R R)$ is calculated by the formula $R R R=(1-(P R B / P R A)) \times 100$, where PRB is the probability of decreasing POV with drug $B$ and PRA the probability of decreasing POV with drug $A$. Relative risk reduction is expressed in percentage ${ }^{6,7}$. In our study, RRR $=72 \%$ when dexamethasone was compared to metoclopramide during the first 4 postoperative hours, expresses that dexamethasone is $72 \%$ more effective as compared to metoclopramide. The number necessary to treat (NNT) is obtained by the formula NNT=1/(PEA-POT), where PEA is the expected probability of the adverse event and POT is the observed probability in the treated group ${ }^{6,7}$. In our study, dexamethasone in the first 4 postoperative hours had NNT $=3.25$, better than metoclopramide (NNT = 15.66), meaning that with dexamethasone, 3.25 patients have to be treated to prevent one POV episode, while with metoclopramide, 15.66 patients have to be treated to prevent one POV episode.

The understanding of POV studies has improved after the concepts of relative risk reduction and number necessary to treat were expanded to this field of study. This has determined the number of patients to be treated to obtain a desired effect. The advantage of this approximation is to allow indirect comparisons of several therapeutic regimens. These mathematical models are not opposed to traditional statistical tests, but rather add to them ${ }^{6-8}$.

POV is classically described in the literature as an obstacle to early discharge, which is exactly the aim of ambulatory anesthesia ${ }^{1,10}$. In our study, however, there was no statistically significant difference between groups in time between surgery completion and hospital discharge, and there was no hospital admission due to incoercible vomiting.

This study has concluded that dexamethasone was more effective than metoclopramide in decreasing the incidence of postoperative vomiting, when used during anesthetic induction with sevoflurane associated to nitrous oxide and fentanyl, in male children submitted to ambulatory inguinal hernia correction 


\section{REFERÊNCIAS - REFERENCES}

01. Watcha MF - Management of postoperative vomiting in pediatric patients. Cur Op Anaesth, 2003;16:575-583.

02. Olutoye $\mathrm{O}$, Watcha MF - Management of postoperative vomiting in pediatric patients. Int Anesthesiol Clin, 2003;41:99-117.

03. Scuderi PE - Pharmacology of antiemetics. Int Anesthesiol Clin, 2003;41:41-66.

04. Gan TJ, Meyer T, Apfel CC et al - Consensus guidelines for managing postoperative nausea and vomiting. Anesth Analg, 2003;97:62-71

05. Carvalho WA, Vianna PTG, Braz JRC - Náuseas e vômitos em anestesia: fisiopatologia e tratamento. Rev Bras Anestesiol, 1999;49:65-79.

06. Schulman SR, Schardt C, Erb T - Evidence-based medicine in anesthesiology. Cur Op Anaesthesiol, 2002;15:661-668.

07. Angelis RMC, Avezum Jr A, Cavalcanti AB et al - Anestesiologia baseada em evidências. O que é e como praticar. Rev Bras Anestesiol, 2004;54:582-594.

08. Duval Neto GF - Anestesiologia na era da medicina baseada em evidências. Rev Bras Anestesiol, 2004;54:141-144.

09. Apfel CC, Roewer N, Korttila K - How to study postoperative nausea and vomiting. Acta Anaesthesiol Scand, 2002;46:921-928.

10. Scuderi PE, Conlay LA - Postoperative nausea and vomiting and outcome. Int Anesthesiol Clin, 2003;41:165-174.

11. Apfel CC, Roewer $N$ - Risk assessment of postoperative nausea and vomiting. Int Anesthesiol Clin, 2003;41:13-32.

12. Eberhart LHJ, Morin AM, Guber D et al - Applicability of risk scores for postoperative nausea and vomiting in adults to paediatric patients. Br J Anaesth, 2004;93:386-392.

13. Everett LL - Can the risk of postoperative nausea and vomiting be identified and lowered during the preoperative assessment? Int Anesthesiol Clin, 2002;40:47-62.

14. Villeret I, Laffon M, Duchalais A et al - Incidence of postoperative nausea and vomiting in paediatric ambulatory surgery. Paediatr Anaesth, 2002;12:712-717.

15. Henzi I, Walder B, Tramer MR - Metoclopramide in the prevention of postoperative nausea and vomiting: a quantitative systematic review of randomized, placebo-controlled studies. $\mathrm{Br} \mathrm{J}$ Anaesth, 1999:83:761-771.

16. Henzi I, Walder B, Tramer MR - Dexamethasone for the prevention of postoperative nausea and vomiting: a quantitative systematic review. Anesth Analg, 2000;20:186-194

\section{RESUMEN}

Bedin A, Pinho MSL, Zanotelli CT, Caldart AS, Turazzi JC, Castro RAC - Dexametasona Comparada a la Metoclopramida en la Prevención de Vómitos Pos-Operatorios en Niños Sometidos a Procedimientos Quirúrgicos Ambulatoriales

JUSTIFICATIVA Y OBJETIVOS: El vómito pos-operatorio es una complicación común y desagradable. Sin embargo, actualmente modelos matemáticos tales como número necesario para tratar (NNT) y reducción del riesgo relativo (RRR) han sido útiles para la decisión de cual medicación utilizar para la profilaxis. El objetivo de este estudio fue verificar si la dexametasona, comparada a la metoclopramida, reduce la incidencia de vómitos cuando administrada por vía venosa en niños anestesiados con sevoflurano en cirugías pediátricas ambulatoriales.

MÉTODO: Participaron del estudio 237 niños, del sexo masculino, con edad entre 11 meses y 12 años, estado físico ASA I y II, sometidos a herniorrafia inguinal. Como medicación pre-anestésica fue utilizado midazolan por vía oral. Para la inducción y mantenimiento de la anestesia fue utilizado sevoflurano, óxido nitroso y fentanil $\left(1 \mu \mathrm{g} . \mathrm{kg}^{-1}\right)$. Los pacientes fueron divididos en grupo $D(n=118)$ dexametasona (150 $\left.\mu \mathrm{g} . \mathrm{kg}^{-1}\right)$ por vía venosa en la inducción y grupo $M(n=119)$ metoclopramida (150 $\left.\mu \mathrm{g} . \mathrm{kg}^{-1}\right)$ en la inducción. Fueron analizadas las incidencias de vómitos en las primeras 4 horas de pos-operatorio (PO), entre 4 horas y 24 horas de $P O$, el NNT de ambas medicaciones utilizadas y la $R R R$ de la dexametasona con relación a la metoclopramida.

RESULTADOS: La incidencia de vómitos fue 9,32\% en el grupo $D$ y $33,61 \%$ en el grupo $M$ durante las primeras 4 horas de $P O$ y $1,69 \%$ con la dexametasona y del 3,36\% con la metoclopramida entre 4 y 24 horas de PO. EI RRR fue $72 \%$ de la dexametasona con relación a la metoclopramida en las primeras 4 horas. El NNT de la dexametasona fue 3,25 y de la metoclopramida fue 15,66.

CONCLUSIONES: La dexametasona reduce, de forma más eficiente que la metoclopramida, la incidencia de vómitos cuando utilizada durante la inducción de anestesia con sevoflurano asociado al óxido nitroso y fentanil. 